\title{
Cataract Surgery for Tilted Lens in Peters' Anomaly Type 2
}

\author{
Tadayuki Nishide Misako Nakanishi Natsuki Hayakawa Ikuko Kimura \\ Nobuhisa Mizuki
}

Department of Ophthalmology and Visual Science, Yokohama City University Graduate School of Medicine, Yokohama City, Japan

\section{Key Words}

Peters' anomaly type $2 \cdot$ Synechia $\cdot$ Phacoemulsification $\cdot$ Tilted lens

\begin{abstract}
Background: Cases of cataract surgery without penetrating keratoplasty in patients with Peters' anomaly are very rare. We report a case of Peters' anomaly type 2 with tilted lens due to synechia between the lens and iris that was treated with cataract surgery without penetrating keratoplasty. Case Presentation: A 16-year-old girl had Peters' anomaly in both eyes. Corneal opacity was severe in the left eye due to high-grade dysgenesis of the anterior segment. In the right eye, corneal opacity had spread from the center of the cornea to the inferotemporal side, and there was synechia between the iris and corneal endothelium from the inferonasal side to the inferotemporal side. Opacity was observed in the anterior pole of the lens, and there was synechia between the anterior iris and the lens. Ultrasound biomicroscopy (UBM) revealed that the lens was tilted because of synechia. The tilted lens induced astigmatism, which reduced visual acuity to $20 / 250$, in conjunction with a cataract. Cataract surgery was performed; the synechia between the lens capsule and the iris was severed, an intraocular lens was inserted, and the tilt was repaired. UBM was used postoperatively to confirm that the lens capsule synechia had been corrected and that the intraocular lens was not tilted. As a result, visual acuity improved to 20/100; glaucoma and expansion of corneal opacity were not observed. Conclusions: Severing of the synechia between the cataract and iris, during cataract surgery, in a patient with Peters' anomaly type 2 resulted in favorable postoperative visual acuity.


Nishide et al.: Cataract Surgery for Tilted Lens in Peters' Anomaly Type 2

\section{Background}

Peters' anomaly cases without cataracts are referred to as type 1, whereas those with cataracts are type 2 [1]. This condition involves dysgenesis of the anterior segment of the eye and occurs more frequently than other conditions that cause anterior segment dysgenesis [2]. In Peters' anomaly, opacity and edema are observed in the center of the cornea from birth, and anomalies are observed in both eyes in $67.2 \%$ of all cases [3].

Peters' anomaly is believed to result from the failure of the lens to separate from the surface ectoderm, which forms the cornea, iris, and angle, during embryogenesis [4]. Agenesis of corneal endothelial cells and Descemet's membrane is observed, as is synechia between the corneal endothelium and the iris. Severe corneal opacity requires penetrating keratoplasty; the prognosis was often poor before [5-8]. Visual acuity is sometimes favorable in cases in which the corneal opacity and cataracts are localized. However, reports of cataract surgery without penetrating keratoplasty in Peters' anomaly type 2 are rare [4, 9].

\section{Case Presentation}

A 16-year-old female, born with corneal opacity in both eyes, developed cataracts and was diagnosed with Peters' anomaly type 2 . This patient did not exhibit any of the systemic abnormalities commonly associated with Peters' anomaly. Corneal opacity and conjunctival epithelial flaps had been observed in the left eye since childhood due to the high-grade dysgenesis of the anterior segment, and light perception was absent in the left eye. In the right eye, the corneal opacity had spread from the center of the cornea to the inferotemporal side (fig. 1a), and a synechia was detected between the corneal epithelium and the iris from the inferonasal to the inferotemporal side. Additionally, preoperative changes were detected in the circle form of the inferotemporal pupillary margin, due to the synechia (fig. 1a). Severe opacity was also observed in the cornea overlying the inferotemporal synechia between the corneal endothelium and the iris. Fortunately, the lens was still visible, because the central cornea had only mild opacification. A cataract was present in the lens, with severe opacity at the anterior pole. Ultrasound biomicroscopy (UBM) of the anterior segment revealed synechia between the anterior iris and the lens; an anterior deviation and tilting of the lens were also observed (fig. 1b). The right eye had an axial length of $27.39 \mathrm{~mm}$, with a preoperative visual acuity of 20/250 and a preoperative refractive error of $-13.00-5.00 \times 120$. The tilted lens resulted in severe astigmatism and was a cause of the diminished visual function. Severe opacity of the lens was observed at the site of the inferotemporal synechia; therefore, cataract surgery was necessary to remove the lens opacity and improve the astigmatism.

The progressive decline in visual acuity caused by the cataract resulted in cataract surgery on the right eye. In order to make synechiolysis from the opposite side, a 2.8-mm clear corneal incision was made on the superonasal side, and viscoelastic materials (Viscoat 0.5, Alcon, Tokyo, Japan) were used to protect the corneal epithelium and maintain the intracameral space. The inferonasal synechia was severed between the cornea and the iris using intraocular scissors. The synechia between the anterior iris and the lens capsule was also severed with intraocular scissors. Phacoemulsification was performed and an intraocular lens (16.5 diopter, KS-Xs, spherical lens, STAAR, Chiba, Japan) was inserted into the capsular bag.

Neither hemorrhage nor increased intraocular pressure were observed postoperatively. Six months postoperatively, there had not been any positional deviation of the intraocular 
Nishide et al.: Cataract Surgery for Tilted Lens in Peters' Anomaly Type 2

lens, nor increase in corneal opacity (fig. 2a); UBM did not reveal any positional deviation of the intraocular lens (fig. 2b). At this time, the patient's visual acuity had improved to 20/100, with a postoperative refractive error of $+0.50-2.00 \times 60$. The large improvement in astigmatism was due to the absence of any intraocular lens positional deviations.

\section{Discussion}

In many cases of Peters' anomaly, penetrating keratoplasty is performed due to the opacity in the center of the cornea, and glaucoma surgery is performed due to angle dysgenesis with increased intraocular pressure. However, when these abnormalities are mild, cataracts become the primary cause of diminished visual acuity. Cataracts cause declines in visual acuity resulting in the need for cataract surgery to improve visual function.

In this case, there were two synechiae between the corneal endothelium and the iris, on the inferonasal and inferotemporal sides of the anterior chamber. The inferonasal synechia was mild. The severing of the iris slightly below the synechia may have increased the flexibility of the iris, making the pupillary margin cylindrical in the non-synechial part of the iris, postoperatively. On the other hand, the inferotemporal synechia was extensive and firm; an attempt to remove the synechia could have expanded the corneal endothelial damage and increased the postoperative hemorrhage of the iris and the corneal opacity. Therefore, we elected not to remove that synechia. We performed cataract surgery using slit lamp to reduce light refraction by corneal opacity, and we did not use trypan blue and papillary expanders to prevent endothelial damage and hemorrhage of the iris.

When performing cataract surgery, a clear corneal incision should be used rather than a corneoscleral incision in order to preserve the conjunctivae. Intact conjunctivae have good influence to trabeculectomy if it is necessary. Cataract surgery was thought likely to induce corneal astigmatism, in this case, and the clear corneal incision was performed on the superonasal side. This site was contralateral to the site of the synechia between the anterior iris and lens capsule, and allowed the synechia to be severed without the intraoperative use of special instruments or the creation of surgical wounds.

In summary, cataract surgery was performed to correct a tilted lens in a patient with Peters' anomaly type 2 . Removal of the opacity and the tilt in the lens resulted in reduced astigmatism and improved visual function. We believe that cataract surgery is desirable in cases with severe refractive error and in cases in which a tilted lens exists.

\section{Acknowledgements}

I would like to express my great appreciation to Professor Nobuhisa Mizuki for his valuable and constructive suggestions during the planning and development of this research work.

\section{References}

1 Zaidman GW, Flanagan JK, Furey CC: Long-term visual prognosis in children after corneal transplant surgery for Peters anomaly type I. Am J Ophthalmol 2007;144:104-108.

2 Shigeyasu C, Yamada M, Mizuno Y, Yokoi T, Nishina S, Azuma N: Clinical features of anterior segment dysgenesis associated with congenital corneal opacities. Cornea 2012;31:293-298.

3 Bhandari R, Ferri S, Whittaker B, Liu M, Lazzaro DR: Peters anomaly: review of the literature. Cornea 2011;30:939-944. 


\begin{tabular}{l|l}
\hline Case Rep Ophthalmol 2013:4:134-137 \\
\hline DOI: 10.1159/000354611 & $\begin{array}{l}\text { ○ } 2013 \text { S. Karger AG, Basel } \\
\text { www.karger.com/cop }\end{array}$ \\
\hline
\end{tabular}

Nishide et al.: Cataract Surgery for Tilted Lens in Peters' Anomaly Type 2

4 Withers SJ, Gole GA, Summers KM: Autosomal dominant cataracts and Peters anomaly in a large Australian family. Clin Genet 1999;55:240-247.

5 Yang LL, Lambert SR: Peters' anomaly. A synopsis of surgical management and visual outcome. Ophthalmol Clin North Am 2001;14:467-477.

-6 Yang LL, Lambert SR, Lynn MJ, Stulting RD: Long-term results of corneal graft survival in infants and children with Peters anomaly. Ophthalmology 1999;106:833-848.

7 Rao KV, Fernandes M, Gangopadhyay N, Vemuganti GK, Krishnaiah S, Sangwan VS: Outcome of penetrating keratoplasty for Peters anomaly. Cornea 2008;27:749-753.

-8 Parmley VC, Stonecipher KG, Rowsey JJ: Peters' anomaly: a review of 26 penetrating keratoplasties in infants. Ophthalmic Surg 1993;24:31-35.

9 Salmon JF, Wallis CE, Murray AD: Variable expressivity of autosomal dominant microcornea with cataract. Arch Ophthalmol 1988;106:505-510.
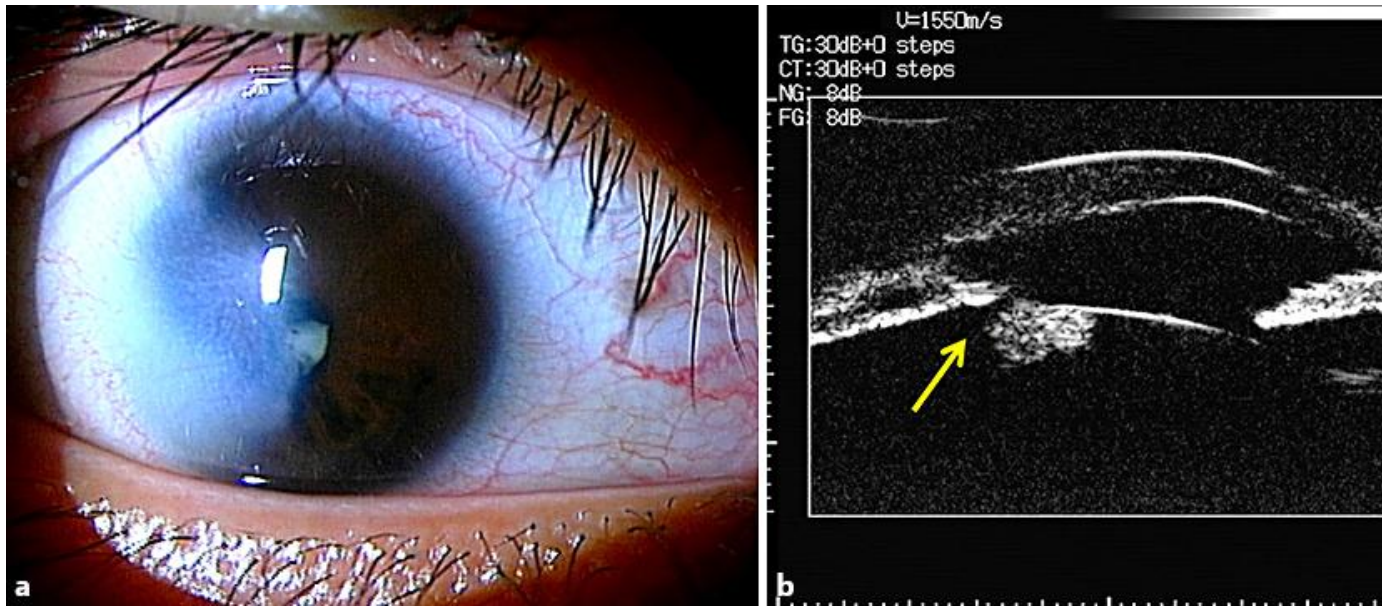

Fig. 1. a Preoperative photo of the anterior segment. Mild corneal opacity and lens opacity in the anterior pole are observed. The circle form of the pupillary margin is seen to be changing on the inferotemporal side. b Preoperative UBM image. The arrow shows severe inferotemporal synechia between the corneal endothelium and the iris. Corneal edema, overlying the synechia, is also apparent.
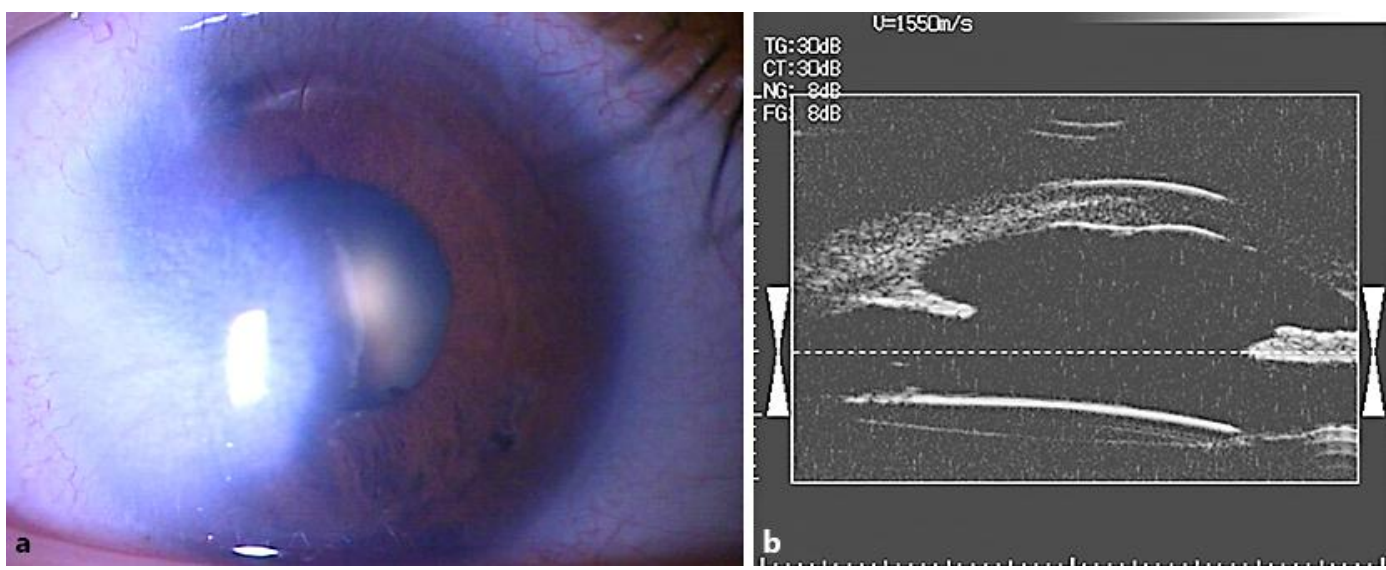

Fig. 2. a Photo of the anterior segment 6 months postoperatively. There is no expansion of the corneal opacity, and the pupil in the non-synechia area is cylindrical. b Postoperative UBM image. No synechiae are observed between the lens capsule and the iris, and the intraocular lens is parallel to the iris plane. 